\title{
Langzeitverhalten von Talsperrenbeton
}

\begin{abstract}
Zusammenfassung: Die Eigenschaften von Sperrenbeton verändern sich mit zunehmendem Alter abhängig von der verwendeten Betonzusammensetzung sowie den Umwelteinflüssen die auf die Talsperre einwirken. Die Festigkeitseigenschaften des Sperrenbetons, wie Druckund Zugfestigkeit sowie Elastizitätsmodul, zeigen mit zunehmendem Betonalter deutlich ansteigende Werte. Im Bezug auf die Dauerhaftigkeit von Talsperrenbeton ist die Widerstandsfähigkeit gegen physikalische und chemische Einwirkungen maßgebend. Global betrachtet ist die Dauerhaftigkeit von Talsperrenbeton am meisten durch einen inneren chemischen Angriff(Alkali-Zuschlag-Reaktion) gefährdet. Aufgrund der verwendeten Betonausgangsstoffe ist, nach derzeitigem Stand der Forschung, eine derartige Gefährdung für österreichische Talsperren nicht gegeben. Schäden an österreichischem Talsperrenbeton sind hauptsächlich auf Frosteinwirkung zurückzuführen, wobei die auftretenden Schäden örtlich begrenzt und für die Standsicherheit der Talsperren nicht maßgebend sind.
\end{abstract}

Long-term concrete behaviour in large dams

Summary: The properties of dam concrete tend to change along with increasing age as a function of concrete composition and the effects of the natural forces acting on the structure. The strength properties of dam concrete, such as compressive and tensile strengths and modulus of elasticity, clearly show rising levels along with increasing concrete age. The durability of dam concrete is determined by its resistance against physical and chemical agents. In global terms, the greatest hazard for the durability of dam concrete is from internal chemical attack (alkali-aggregate reaction). The latest research results suggest, however, that no such hazard exists for Austrian dams, thanks to the raw materials used for the concrete. Most of the damage to Austrian dams is caused by frost, but this is localised in area and has no bearing on the stability of the structure.

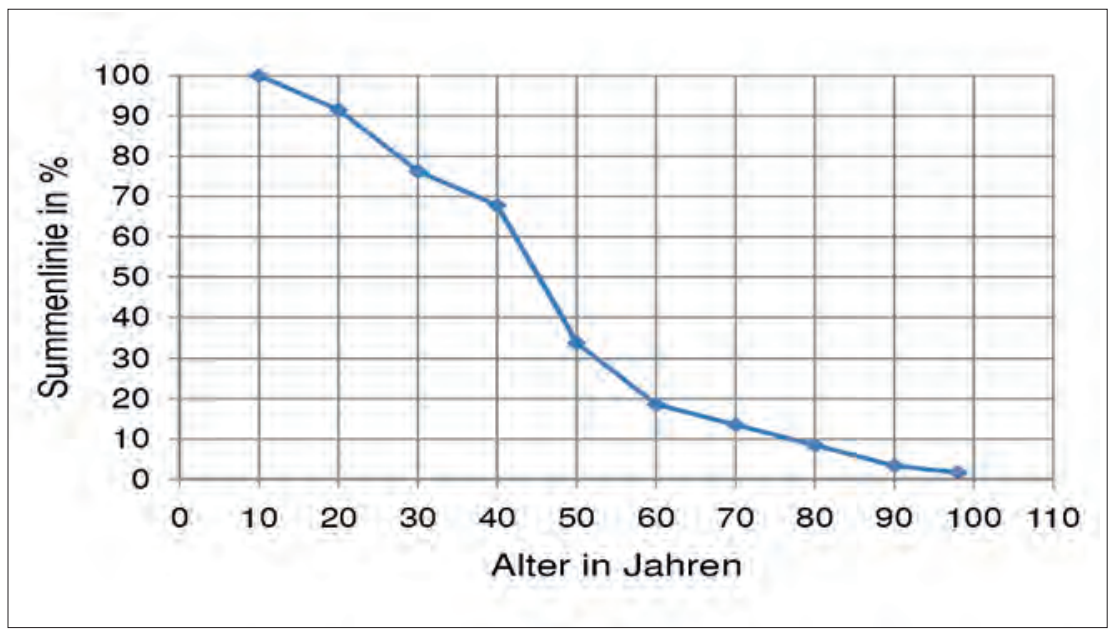

Abb. 1: Alter österreichischer Talsperren in \% der gesamten Anlagen.

\section{Allgemein}

Die Geschichte des Talsperrenbaues reicht mindestens 5000 Jahre zurück. Als eine der ersten Gewölbemauer der Welt gilt die Talsperre Vallon de Baume (Höhe $12 \mathrm{~m}$ ) in Frankreich die in der Römerzeit errichtet wurde. Die ersten Gewölbemauern seit der Römerzeit dürfte der Kerba Dam (ca. 1300) und der Kurit Dam (ca. 1350, Höhe $60 \mathrm{~m}$ ), beide im Iran von den Mongolen errichtet, sein. Als die erste nach neuzeitlichen Gesichtspunkten errichtete Talsperre wird die Gewölbemauer „Elche“ (1640) in Spanien genannt.

Infrastrukturbauwerke werden in der Regel rechnerisch für eine „Lebensdauer “ von 100 Jahren ausgelegt. Tatsächlich ist die Lebensdauer der Talsperren bedeutend länger. Bauwerke wie die Kölnbreinsperre, die Zillergründlsperre usw. werden weit über 100 Jahre in Betrieb bleiben.

Eine Auflistung (lit. ATCOLD) der Österreichischen Talsperren im Bezug auf die Anzahl und das Alter der Talsperren ist im Abbildung 1.

Die ersten österreichischen Talsperren erreichen in 2 bis 3 Jahren ein Alter von 100 Jahren (Sperre Erlaufsee und Sperre Wiestal). Mehr als 30\% der österreichischen Talsperren sind älter als 50 Jahre.

Beton ist ein sehr robuster Baustoff, der allerdings einem gewissen Alterungsprozess unterliegt. Das Langzeitverhaltens des Baustoffes Beton wird in der Folge in Hinblick auf die für die Tragsicherheit sowie der Dauerhaftigkeit der Talsperren maßgebenden Parametern analysiert.

\section{Festigkeitsentwicklung}

Für die Festigkeitsentwicklung des Sperrebetons über Jahre und Jahrzehnte ist das verwendete Bindemittel von maßgebender Bedeutung.

Für die in Österreich gebauten Talsperren wurde Portlandzement verwendet. Diesem wurde am Beginn des 19. Jahrhunderts Trass oder hydraulischer Kalk (Romanzement) beigemengt (z. B. Sperre Wiestal). In späterer Folge wurde beispielsweise bei den Sperren Drossen, Mooser, Limberg (2), Spullersee oder Tauernmoos Portlandzement mit geringer Mahlfeinheit ohne $\mathrm{Zu}$ mahlstoffe verwendet. Für die Sperre Schlegeis (1969 bis 1971) wurde ein Sonderzement (Hochofenzement mit einem Schlackegehalt von 50-55\%) (3) zur Reduktion der Hydratationswärme für den Sperrenbeton eingesetzt. Für die Talsperren jüngerer Bauart (Kölnbrein, Zillergründl) wurde Portlandzement mit Flugasche (bis $33 \%)(4,6)$ verwendet.

\subsection{Druckfestigkeit}

Das Prüfalter für den Nachweis der Druckfestigkeit bezog sich für Sperrenbe- 


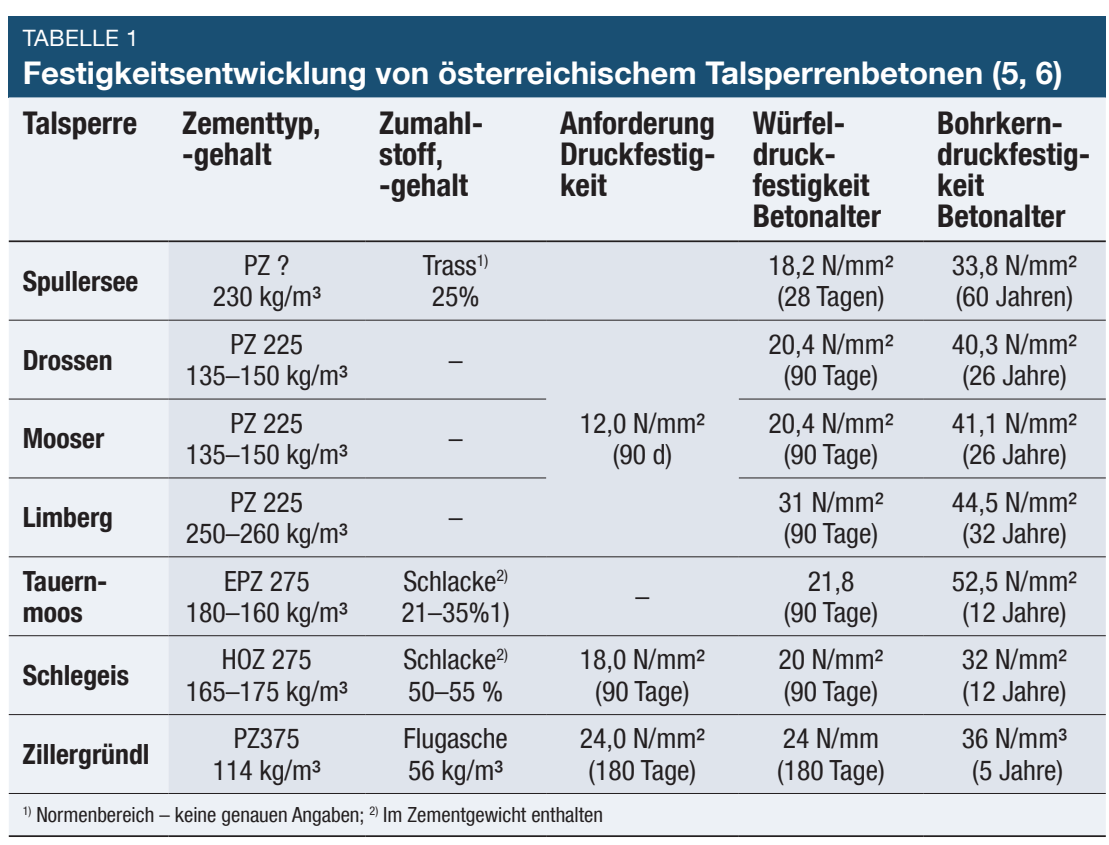

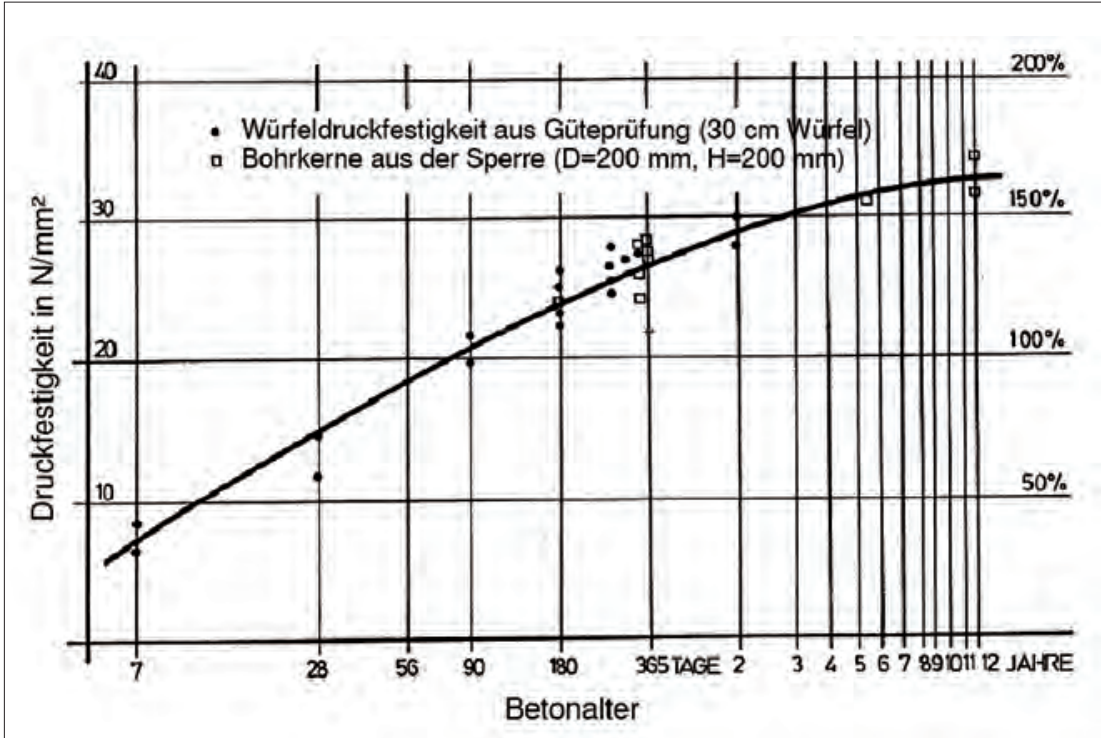

Abb. 2: Druckfestigkeitsentwicklung des Betons der Sperre Schlegeis (5)

ton auf 28 oder 90 Tage (ältere Sperren) bzw. bis 180 Tage (1) (jüngere Sperrenbauten). Aufgrund der geringen Mahlfeinheit des Zementes oder der Zugabe von puzzolanischen Stoffen wie Schlacke oder Flugasche erfolgt eine weitere Festigkeitsentwicklung über Jahre und Jahrzehnte.

In Tabelle 1 sind die Festigkeitsentwicklungen unterschiedlicher österreichischer Talsperrenbetone enthalten. Die Festigkeiten bei der Bauausführung sind als Würfeldruckfestigkeiten $(30 \mathrm{~cm}$ Würfel) angegeben. Die Druckfestigkeiten im Bauwerk wurden an Bohrkernen mit einem Durchmesser von $200 \mathrm{~mm}$ und Höhe von $200 \mathrm{~mm}$ bestimmt. Die angegebenen Werte für die Druckfestigkeit sind Mittelwerte.

Aus den Ergebnissen der Tabelle 1 geht hervor, dass sich die Druckfestigkeit des Sperrenbetons, abhängig vom Bindemittel und dem Prüfalter um 50-100\% gegenüber den 28, 90 bzw. 180 Tage Werten, im Laufe der Jahre und Jahrzehnte erhöht hat.

Im folgenden Abbildung 1 ist die Festigkeitsentwicklung des Sperrenbetons der Sperre Schleigeis über einen Zeitraum von 12 Jahren anhand der Güteprüfung an gesondert hergestellten Proben $(30 \mathrm{~cm}$ Würfel) und an Bohrkernen aus dem Bauwerk dargestellt.
Gegenüber der geforderten Festigkeit von $18 \mathrm{~N} / \mathrm{mm}^{2}$ hat sich die Druckfestigkeit des Betons der Sperre Schlegeis im Laufe von 12 Jahren fast verdoppelt.

\subsection{Zugfestigkeit}

Für die Entwicklung der Zugfestigkeit von Sperrenbeton über Jahre und Jahrzehnte liegen nur eingeschränkt Ergebnisse vor. Für die vorliegenden Ergebnisse ist zu berücksichtigen, dass aufgrund des geringen Probendurchmessers $(200 \mathrm{~mm})$ im Verhältnis zum Größtkorn im Beton (bis $150 \mathrm{~mm}$ ) die Ergebnisse mit einer hohen Prüfstreuung versehen sind.

In der Tabelle 2 sind die Ergebnisse der Prüfung der Spaltzugfestigkeit von Sperrenbetonen (Druckfestigkeit siehe Tabelle 1) enthalten.

Gemäß einer theoretischen Berechnung der zu erwartenden Betonzugfestigkeit auf der Basis der geforderten Druckfestigkeit der Talsperrenbetone (nach 28, 90 bzw. 180 Tagen) gemäß (7) zeigt, dass die ermittelten Spaltzugfestigkeiten an Bohrkernen der einzelnen Sperrenbetone die nach Jahren entnommen wurden um den Faktor 1,7 bis 1,9 höher sind.

In Abbildung 3 ist eine Gegenüberstellung der ermittelten Spaltzugfestigkeit verschiedener Sperrenbetone gegenüber der theoretischen Spaltzugfestigkeit, errechnet nach (7) und den tatsächlich ermittelten Werten aus Tabelle 2.

Eine Gegenüberstellung der Ergebnisse der Prüfung der Spaltzugfestigkeit an Bohrkernen gegenüber der theoretischen Berechnung (7) der Spaltzugfestigkeit aus der geprüften Betondruckfestigkeit zeigt keine Korrelation. Die theoretische Berechnung der Spaltzugfestigkeit auf Basis der Betondruckfestigkeit beruht auf der Prüfung von Laborproben. In (7) wird darauf hingewiesen, dass die Spaltzugfestigkeit die an Proben aus dem Sperrenbeton ermittelt wird keine Korrelation mit Ergebnissen aus Laborproben ermöglicht. Diese Feststellung kann anhand der vorliegenden Prüfergebnisse bestätigt werden.

\subsection{Moduli}

Talsperrenbeton durchläuft im Bezug auf das Langzeitverhalten des Betons 3 Phasen der Entwicklung des E-Moduls. Beim ersten Einstau wirkt der Erstverformungsmodul, der geringer ist als der statische E-Modul(Abbildung 4). Nach2 bis3 Lastwechseln stellt sich der statische E-Modul ein. Mit 


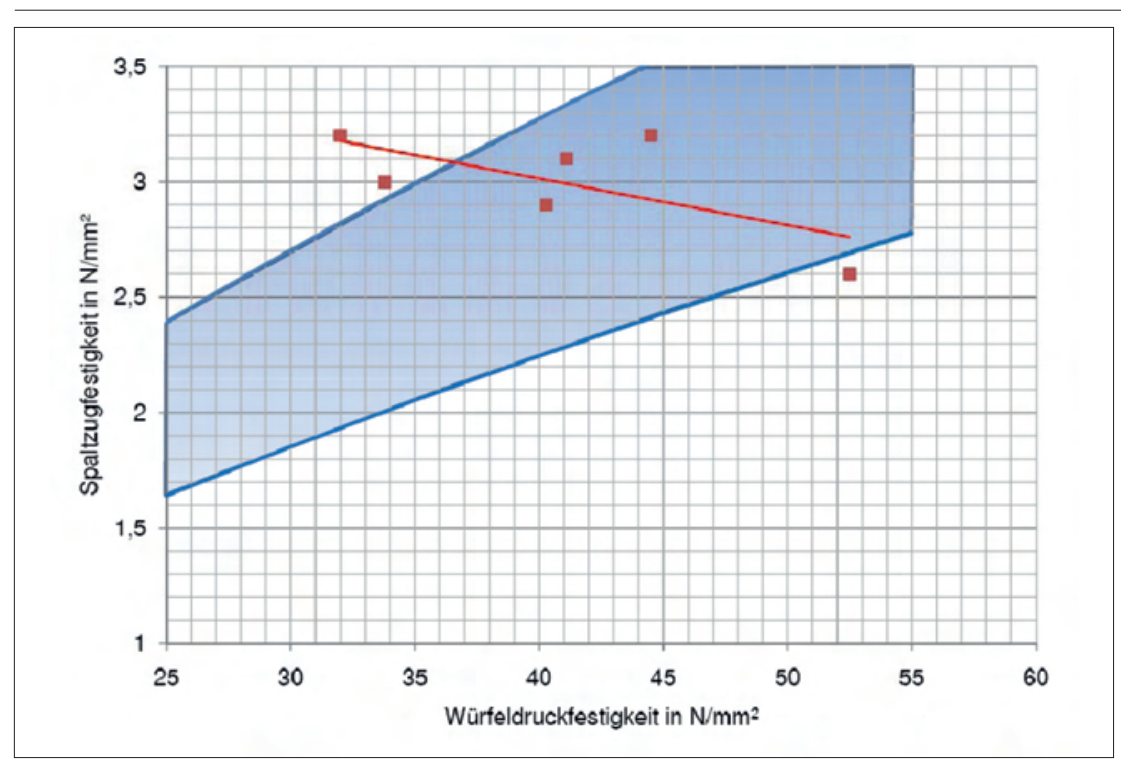

Abb. 3: Spaltzugfestigkeit bestimmt an Bohrkernen (Rot) gegenüber der theoretischen Spaltzugfestigkeit gemäß (7).

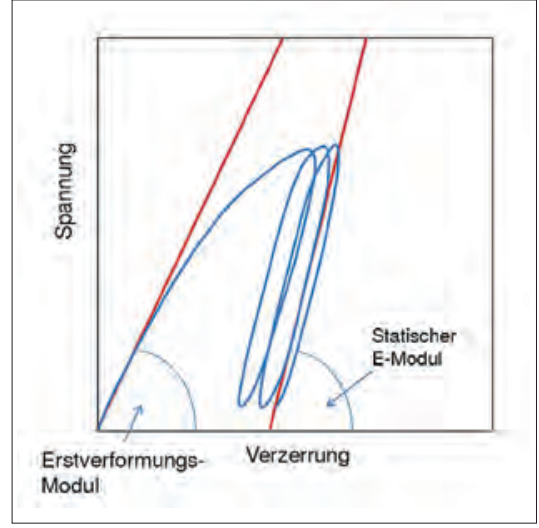

Abb. 4: Verschiedene Formen des E-Modul.

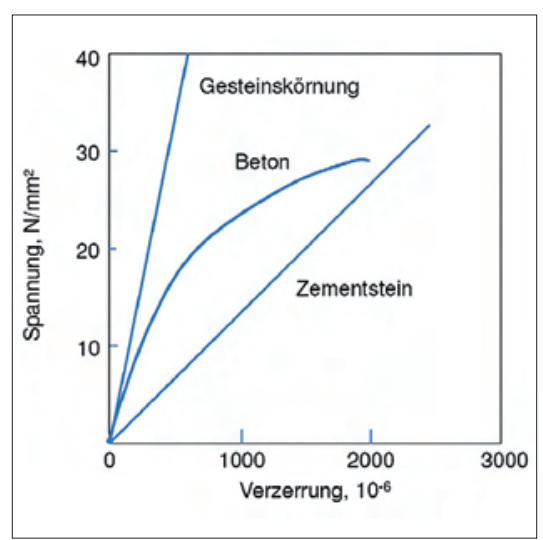

Abb. 5: E-Moduli des Gesteinskörnung, des Zementsteins und des Betons (7).

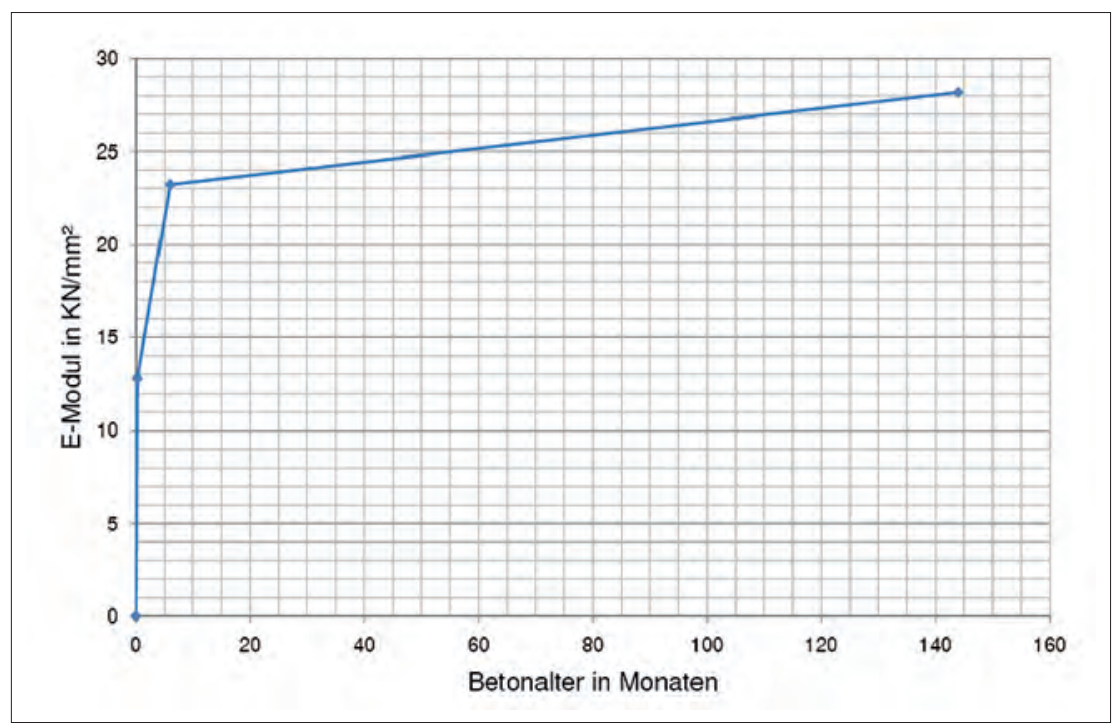

Abb. 6: Entwicklung des E-Moduls des Betons der Sperre Schlegeis. zunehmendem Betonalter steigt der statische E-Modul infolge der fortlaufenden Hydratation des Bindemittels weiter an.

Durch die über Jahre dauernde Hydratation des Bindemittels steigt der E-Modul des Zementsteins wodurch sich der E-Modul des Betons erhöht. In der Regel ist der E-Modul der Gesteinskörnung höher als der E-Modul des Zementsteins wodurch der E-Modul des Betons mit der Höhe des E-Moduls der Gesteinskörnung begrenzt ist (Abb. 5).

In Tabelle 3 sind die Ergebnisse der ermittelten Werte für den E-Modul, bestimmt an Bohrkernen aus den Talsperren den Ergebnissen des E-Moduls der während des Sperrenbaues ermittelt wurde (Güteprüfung) gegenübergestellt.

In Abbildung 6 ist die Entwicklung des E-Moduls des Betons der Sperre Schlegeis ersichtlich. Mehr als $80 \%$ des E-Moduls der nach 12 Jahren bestimmt wurde ist bereits bei einem Betonalter von 6 Monaten erreicht.

\section{Dauerhaftigkeit}

Im Bezug auf die Dauerhaftigkeit des Sperrenbetons ist zwischen physikalischem und chemischem Schädigungsmechanismus zu unterscheiden. Als physikalischer Angriff ist der Frostangriff zu nennen. Äußere chemische Schädingungsmechanismus sind aufgrund des Verhältnisses von Betonoberfläche zum Betonvolumen bei entsprechend dichtem Betongefüge von untergeordneter Bedeutung. Innere chemische Schädigungsmechanismen spielen dagegen eine bedeutende Rolle wie es sich in den letzten Jahren anhand der weltweit gemeldeten Schäden durch Alkali-Aggregat Reaktionen zeigte.

\subsection{Wasserdurchlässigkeit}

Wasserdurchlässigkeiten an österreichischen Talsperren erfolgen in der Regel nicht durch das Betongefüge sondern wenn, dann an Fugen zwischen Betonierzonen (Arbeitsfugen) oder an undichten Blockfugen. Eine Ausnahme stellen Talsperren dar die vor ca. 100 Jahren erbaut wurden. Aufgrund der damaligen Einbaumethode für den Beton (Stampfbeton) wurden Inhomogenitäten (Kiesnester) im Betongefüge erzeugt die Wasserwegigkeiten innerhalb der Sperren ermöglichen (Abb. 7).

Derartige Mausgänge oder Inhomogenitäten (Kiesnester) können, wenn sie innerhalb der Sperre durchgehend miteinander verbunden sind, zu punktuellen Feuchtstellen oder Wasseraustritten führen. 
Feuchtstellen oder geringfügige Wasserdurchtritte an Arbeitsfugen oder Blockfugen stellen lediglich im Hinblick auf die Dauerhaftigkeit ein Problem dar. Durch Eisbildung im Winter kann es zu Folgeschäden wie örtliche Betonabplatzungen oder Beschädigung des Fugenbandes kommen.

\subsection{Frostbeständigkeit}

Bei Talsperren die seit ca. 1951 gebaut wurden ist in der Regel Luftporenbildner dem Sperrenbeton zugesetzt worden. Dies stellt im Hinblick auf die Frostbeständigkeit des Sperrenbetons eine deutliche Erhöhung der Dauerhaftigkeit dar.

Frostbeständigkeitsprüfungen von Sperrenbetonproben, die nach Jahren der Betriebsdauer aus den Talsperren Drossen, Mooserboden, Weißsee, Tauernmoos und Schlegeis (alle nach 1951 erstellt) haben die Frostbeständigkeit der verwendeten Betone bestätigt.

Eine Prüfungen der Frostbeständigkeit an Proben der Talsperren Spullersee (errichtet 1922-26), Salza (errichtet 1947-49) und Limberg (errichtet 1948-51) zeigte, dass diese Sperrenbetone gemäß normgemäßer Definition (ÖN B 3303, Ausgabe 1983) nicht frostbeständig sind.

Die Frostprüfung im Laborversuch weist ein äußerst hohes Beanspruchungsniveau auf welches in der Natur kaum erreicht wird. Dies bestätigt sich auch an nur unbedeutende Frostschäden die an den Talsperren erkennbar sind welche keinen normengemäß frostbeständigen Sperrenbeton aufweisen.

Langfristig ist an diesen Sperren allerdings mit einem Auftreten von lokalen

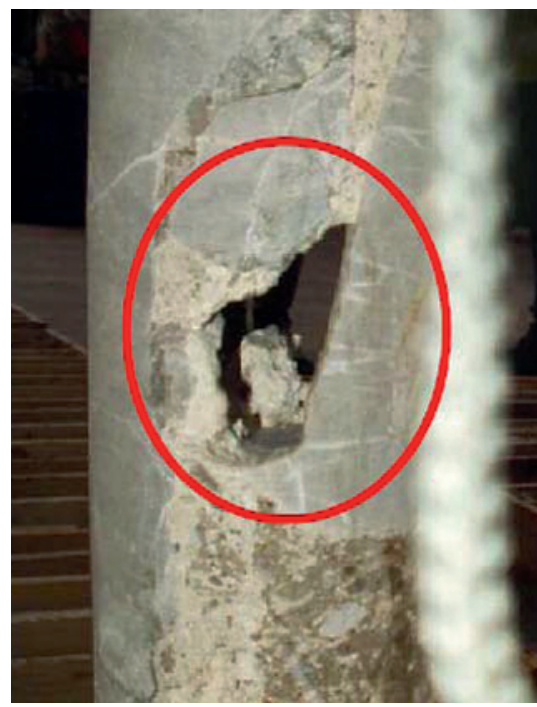

Abb. 7: "Mausgang“. oberflächlichen Schäden (Frostabplatzungen) auf der Wasserseite zu rechnen, wie sie an der Sperre Salza im Rahmen der Rehabilitierung (2008) festgestellt wurden. Betroffen ist dabei die Betonoberfläche der Wasserseite im Bereich des tiefsten Betriebswasserstandes (Abb. 8).

\subsection{Chemische Beständigkeit}

\subsection{1 Äußerer chemischer Angriff}

Nennenswerte Betonschäden an österreichischen Talsperren durch äußeren chemischen Angriff sind nicht bekannt.

\begin{tabular}{|c|c|c|c|c|}
\hline Talsperre & $\begin{array}{l}\text { Zementtyp, } \\
\text {-gehalt }\end{array}$ & $\begin{array}{l}\text { Zumahlstoff, } \\
\text {-gehalt }\end{array}$ & $\begin{array}{l}\text { Theoretisch gefor- } \\
\text { derte } \\
\text { Spaltzugfestigkeit }\end{array}$ & $\begin{array}{l}\text { Bohrkern-spalt- } \\
\text { zugfestigkeit } \\
\text { Betonalter }\end{array}$ \\
\hline Spullersee & $\begin{array}{c}\mathrm{PZ} ? \\
230 \mathrm{~kg} / \mathrm{m}^{3}\end{array}$ & $\begin{array}{l}\text { Trass } 1) \\
25 \% \\
\end{array}$ & $\begin{array}{l}1,6 \mathrm{~N} / \mathrm{mm}^{2} \\
\text { (28 Tagen) }\end{array}$ & $\begin{array}{l}3,0 \mathrm{~N} / \mathrm{mm}^{2} \\
\text { (60 Jahren) }\end{array}$ \\
\hline Drossen & $\begin{array}{c}\text { PZ } 225 \\
135-150 \mathrm{~kg} / \mathrm{m}^{3}\end{array}$ & - & $\begin{array}{c}1,2 \mathrm{~N} / \mathrm{mm}^{2} \\
\text { (90 Tage) }\end{array}$ & $\begin{array}{l}2,9 \mathrm{~N} / \mathrm{mm}^{2} \\
\text { (26 Jahre) }\end{array}$ \\
\hline Mooser & $\begin{array}{c}\text { PZ } 225 \\
135-150 \mathrm{~kg} / \mathrm{m}^{3}\end{array}$ & - & $\begin{array}{c}1,2 \mathrm{~N} / \mathrm{mm}^{2} \\
\text { (90 Tage) }\end{array}$ & $\begin{array}{l}3,1 \mathrm{~N} / \mathrm{mm}^{2} \\
\text { (26 Jahre) }\end{array}$ \\
\hline Limberg & $\begin{array}{c}\text { PZ 225 } \\
250-260 \mathrm{~kg} / \mathrm{m}^{3}\end{array}$ & - & $\begin{array}{c}1,2 \mathrm{~N} / \mathrm{mm}^{2} \\
(90 \text { Tage) }\end{array}$ & $\begin{array}{l}3,2 \mathrm{~N} / \mathrm{mm}^{2} \\
\text { (32 Jahre) }\end{array}$ \\
\hline Tauernmoos & $\begin{array}{c}\text { EPZ 275 } \\
180-160 \mathrm{~kg} / \mathrm{m}^{3}\end{array}$ & $\begin{array}{l}\text { Schlacke2) } \\
21-35 \% 1)\end{array}$ & $\begin{array}{c}1,8 \mathrm{~N} / \mathrm{mm}^{2} \\
\text { (90 Tage) }\end{array}$ & $\begin{array}{l}\text { 2,6 N/mm² } \\
\text { (12 Jahre) }\end{array}$ \\
\hline Schlegeis & $\begin{array}{c}\text { HOZ 275 } \\
165-175 \mathrm{~kg} / \mathrm{m}^{3}\end{array}$ & $\begin{array}{l}\text { Schlacke }{ }^{2)} \\
50-55 \%\end{array}$ & $\begin{array}{c}1,5 \mathrm{~N} / \mathrm{mm}^{2} \\
\text { (90 Tage) }\end{array}$ & $\begin{array}{l}3,2 \mathrm{~N} / \mathrm{mm}^{2} \\
\text { (12 Jahre) }\end{array}$ \\
\hline
\end{tabular}

1) Normbereich; ${ }^{22}$ Im Zementgewicht enthalten

\begin{tabular}{|c|c|c|c|c|}
\hline Talsperre & $\begin{array}{l}\text { Zementtyp, } \\
\text {-gehalt } \\
\mathrm{kg} / \mathrm{m}^{3}\end{array}$ & $\begin{array}{l}\text { Zumahlstoff, } \\
\text {-gehalt }\end{array}$ & $\begin{array}{l}\text { E-Modul } \\
\text { Güteprüfung } \\
\text { kN/mm² }\end{array}$ & $\begin{array}{l}\text { E-Modul } \\
\text { Bohrkern } \\
\text { kN/mm² }\end{array}$ \\
\hline Weißsee & $\begin{array}{c}\mathrm{PZ} 225 \\
300\end{array}$ & - & $\begin{array}{l}18,3 \\
\text { (90d) }\end{array}$ & $\begin{array}{c}28,8 \\
\text { (32 Jahre) }\end{array}$ \\
\hline Tauernmoos & $\begin{array}{l}\text { EPZ 275 } \\
180-160\end{array}$ & $\begin{array}{l}\text { Schlacke } \\
21-35 \%{ }^{11}\end{array}$ & $\begin{array}{l}20,0 \\
(90 d)\end{array}$ & $\begin{array}{c}19,0 \text { (180d) } \\
26,9 \text { (12 Jahre) }\end{array}$ \\
\hline Schlegeis & $\begin{array}{l}\text { HOZ } 275 \\
165-175\end{array}$ & $\begin{array}{l}\text { Schlacke }{ }^{2)} \\
50-55 \%\end{array}$ & $\begin{array}{c}23,2 \\
(180 d)\end{array}$ & $\begin{array}{c}28,2 \\
\text { (12 Jahre) }\end{array}$ \\
\hline
\end{tabular}

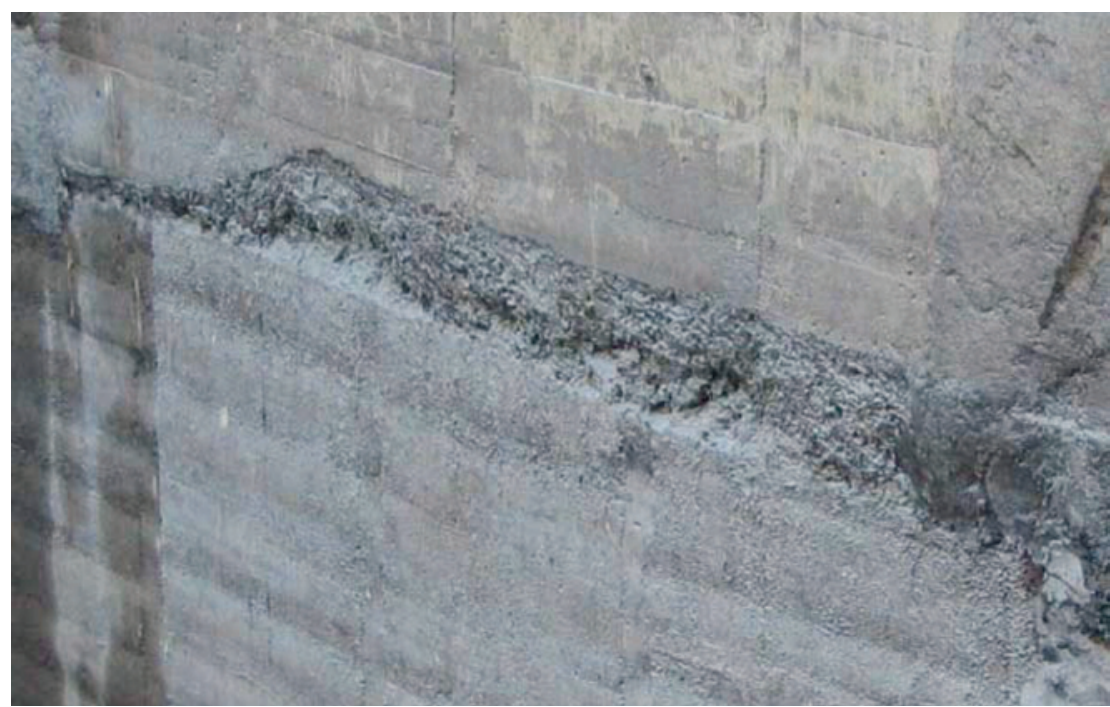

Abb. 8: Oberflächliche Frostschäden an der Wasserseite der Sperre Salza vor der Instandsetzung. 
Durch weiche Wässer, vor allem im Bereich des Alpenhauptkammes, tritt bei einigen Sperren eine geringfügige Lösung des Zementsteins an der wasserseitigen Betonoberfläche auf. Dies führt zu einer waschbetonähnlichen Oberflächenstruktur. Eine Instandsetzung von derartigen Ablösungen ist aufgrund der zu vernachlässigenden Auswirkungen (Betonabtrag von einigen $\mathrm{mm}$ ) nicht erforderlich.

\subsubsection{Innerer chemischer Angriff}

Die weltweit schwerwiegendsten Schäden an Talsperren entstehen aufgrund von schädlichen Alkali-Zuschlag-Reaktionen.

Es sind derzeit 3 Arten von schädlichen Reaktionen von Alkalien und Gesteinskrönungen bekannt:

- Alkali-Kieselsäure-Reaktion

- Alkali-Silikat-Reaktion

- Alkali-Karbonat-Reaktion

In Österreich ist lediglich ein Sperrenbauwerk bekannt an der eine Schädigung durch AAR festgestellt wurde. Die in den Jahren 1943 bis 1945 errichtete Sperre Gmünd (37 m hohe Gewölbemauer) zeigte Deformationen und Rissbildungen, die auf eine AAR zurückzuführen waren. Die Sperre konnte nicht mehr saniert werden und wurde durch eine neu Sperre ersetzt.

Aufgrund der außerhalb Österreichs bekanntgewordenen Schäden wurde unter Federführung der Verbundgesellschaft und unter Teilnahme der Illwerke ein Forschungsprojekt zur Überprüfung von ausgewählten Talsperren in den Jahren 1996-
1998 durchgeführt. An den Abbaufeldern bzw. Zwischendeponien der Gesteinskörnung für die Talsperrenbetone und vom Sperrenbeton selbst wurden Proben gezogen und nach dem damaligen Stand der Wissenschaft geprüft. Ein Potential der Gesteinskörnungen in Hinblick auf eine Alkali-Aggregat-Reaktivität sowie schädliche Alkali-Aggregat-Reaktionen an den Sperrenbetonen konnte dabei nicht festgestellt werden.

\section{Zusammenfassung}

Das Langzeitverhalten der beurteilten österreichischen Talsperrenbetone zeigt qualitativ bewertet eine deutliche Verbesserung der Festigkeitseigenschaften, sowie einen deutlichen Anstieg des E-Moduls.

Alterungserscheinungen im Hinblick auf physikalische Einwirkungen beschränken sich auf oberflächennahe Frostschäden die in der Regel keine Auswirkungen auf die Gebrauchstauglichkeit des Bau- werkes haben. Betroffen von derartigen Schäden, sind fast nur Talsperren die vor ca. 1951 errichtet wurden. Frostschäden an Talsperren die nach 1951 errichtet wurden beschränken sich hauptsächlich auf den Bereich von undichten Arbeits- oder Blockfugen.

Langfristig ist eine schädigende AlkaliAggregat Reaktion die größte Gefahr für den Sperrenbeton. Da diese einen „inneren chemischen Angriff" darstellt sind die Möglichkeiten einer Sanierung praktisch nicht gegeben.

Mit Ausnahme eines Einzelfalles, einer kleinen Talsperre (Gmünd) die berteits erneuert wurde, konnte an österreichischen Talsperrenein derartiges Phänomen bisher nicht festgestellt werden.

\section{Korrespondenz:}

Dipl.-Ing. Dr. techn. Walter Pichler

Material Consult

Niederhart 116a

A-6263 Hart

E-Mail: material.consult@aon.at

\section{LITERATUR}

1 Widmann R (1999) Gewölbemauern, Erfahrungen-Probleme-Entwicklungen, Österreichi(1997) Di Tu Huber H (1997) Die Technik der Osterreich schen Talsperrenbetone, BFT Betonwerk Ferigtil-Technik, Jg: 63, Nr. 10, S66-73 3 Rienössl K, Huber H Die Zemmkraftwerke technische Probleme bei der Planung und Ausführung

4 Huber H (1988) Bauen mit Flugasche, VGBBautagung, S 63-66
5 Huber $\mathbf{H}$ Langzeitverhalten von österreichischen Talsperren und Flußstauwerken, Die Talsperren Österreichs, Heft 28, ATCOLD, S 14-36

6 Huber H (1979) Kölnbreinsperre (Maltakraftwerke) - Neue Wege in der Technik der Maswerke) - Neue Wege in der Technik der Massenbeton

117-124

7 ICOLD Bulletin The physical properties of hardened conventional concrete in dams, Tab. 2.11.; Icold 